\title{
Investigation of an inverse thermal injection mould design methodology in dependence of the part geometry
}

\author{
C. Hopmann ${ }^{1}$ (D) - J. Gerads ${ }^{1}$ (D) $\cdot$ T. Hohlweck ${ }^{1}$ (D)
}

Received: 31 July 2020 / Accepted: 29 November 2020 / Published online: 18 January 2021

(C) The Author(s) 2021

\begin{abstract}
The production of injection moulded components with low shrinkage and warpage is a constant challenge for manufacturers. The thermal design of the injection mould plays an important role for the achievable quality, especially the placement of the cooling channels. This design is usually based on empirical knowledge of the mould designers. The construction is supported iteratively by injection moulding simulations. In the case of thick-walled plastic optics with big wall thickness jumps, the shrinkage is compensated by injection compression moulding. In this process, the thin-walled areas freeze earlier and the necessary compression pressure introduces stresses into these areas which reduces the optical performance. An adapted cooling channel design can reduce these problems. At the IKV, Institute for Plastics Processing in Industry and Crafts at the RWTH Aachen University, a methodology was developed which inversely calculates the cooling requirement of the moulded part A demand-oriented cooling channel system is derived based on the computed results. The aim of the research projects is to minimise displacement and internal stresses by temperature control of the moulded parts according to the demand. In this paper, the methodology is applied to three different geometries, representing three classical parts for the injection moulding process. Three different quality areas in the mould for the inverse optimisation are defined and investigated. For each geometry the cooling channel designs are then validated in injection moulding simulations based on the results from the thermal optimisation. It can be shown that for different component geometries and thicknesses, different quality areas are advantageous and decrease the maximum warpage of the parts. For thin-walled ribbed components, a 2D approach leads to a $15 \%$ smaller displacement, for components with wall thickness jumps, all investigated quality ranges show no differences in displacement, but a surface in the middle of the part is preferred due to a $3{ }^{\circ} \mathrm{C}$ lower standard deviation of the temperature distribution.
\end{abstract}

Keywords Injection moulding simulation - Thermal optimisation · Warpage reduction · Heat transfer · Thermal mold design · Inverse heat transfer problem

\section{Introduction}

Material-related component shrinkage is one of the greatest challenges of modern plastics processing. This is especially challenging for injection moulded components, which are characterised by a high functional density and increasingly complex component geometries. Customer often place high

\section{J. Gerads}

jonas.gerads@ikv.rwth-aachen.de

T. Hohlweck

tobias.hohlweck@ikv.rwth-aachen.de

1 IKV Institute for Plastics Processing in Industry and Craft at RWTH Aachen University, Seffenter Weg 201, 52074 Aachen, Germany demands on geometrical accuracy on these parts, because they are often used in larger assemblies. In case of complex moulded parts, however, due to large numbers of sliders and parting lines in the mould, it is often not possible to introduce a cooling system adapted to the part, which compromises precision [1]. In addition, the cooling time is the dominating factor regarding cycle influencing the cycle time and thus the productivity of the process. Process optimisation in terms of cycle time and geometric accuracy is therefore only possible by adapting the temperature control system to the cooling requirements of the part [2].

In most moulds, drilled cooling channels are used, which are interconnected to form a complete system by targeted cross-cutting and sealing. Drilled cooling channels are currently the manufacturing process with the highest economic relevance due to their simple manufacturing method [2]. 
However, linear drilled cooling channels constrain the 3Dcooling-channel composition significantly. In contrast, technologies such as Selective Laser Melting (SLM) allow the layer-wise, structured build-up of injection moulds based on metal powder. This process enables the realisation of individually adapted and complex cooling channel layouts due to its extremely high design freedom. However, when cooling channels are placed very close to the cavity wall, the thermal gradients on the cavity wall can increase. Thus, badly placed conformal cooling channels can even increase the resulting part warpage. In addition, the thermal mould design is complicated by the comparatively high shrinkage of semicrystalline thermoplastics, which are often used in technical applications [2]. This shrinkage is a result of internal crystallisation processes in the plastic melt and depends on the local temperature conditions. Uneven shrinkage ultimately leads to the formation of stresses in the material, which can only be compensated by warpage of the component. Particular attention must be paid to avoiding hotspots in the mould. These hot spots lead to locally different shrinkage and thus to unacceptable part warpage. This part warpage means that, for example, assembly dimensions can no longer be maintained. It is possible to reduce the warpage with the aid of a temperature control optimized for uniform component shrinkage. In recent years, a simulation routine has been set up at the IKV, Institute for Plastics Processing, to calculate the heat fluxes in the injection mould around the cavity. Up to now, the quality area has only been analysed for thin-walled plastic parts. To realise an inverse thermal cooling channel design for different specimen, such as thick-walled parts, a volumetric approach was implemented in the methodology. In the following paper, the investigation in different quality areas is presented. They are compared to the existing approach for thinwalled injection moulded parts.

\section{State of the art}

\section{Current state of the art in thermal mould design}

Current thermal mould design is a forward iterative process. The analytical thermal mould design has been used over years to ensure a homogenous cavity temperature on the mould surface. Firstly, based on the local cooling error, a design of the cooling channels is derived in a way to minimise the cooling error and achieving a homogeneous temperature distribution. But digital process simulations using the FEM-method to calculate the temperature distribution inside the mould have substituted this analytical methodology based on pen and pencil. Secondly, the mould designer changes the position of the cooling channels such that hot spots will be minimised. Effectiveness and efficiency of this procedure are heavily dependent on the mould designer's experience $[2,3]$.
This is why current process simulation software such as Sigmasoft (SIGMA Engineering GmbH, Aachen, Germany) or Moldflow (Autodesk Inc., San Rafael, USA) offer an optimisation of the mould design by virtual designs of experiment (DoE) $[4,5]$. This optimisation is based on the degrees of freedom given by the designer. By choosing different geometries or process parameters, the designer can define the optimisation space. Several designs can be calculated automatically one after the other. At the end an optimised design is found based on the given preconditions. It is not assured that it is the global optimum regarding the quality criteria.

In this paper, the inverse thermal mould design is also considered for optical parts. In this particular case, the thermal mould design not only effects part warpage but also the optical quality. Due to high wall thickness differences in plastic lenses, standard cooling channel designs lead to residual stresses in the thin-walled areas of the lens due to early solidification of the plastic melt. As a result, introduced residual stresses can cause birefringence, such that a plastic lens does not focus properly. Therefore, the residual stress leads to a reduction in optical performance from the moulded lens $[6$, 7]. One way to reduce the internal stress is to adapt the cooling conditions according to the geometry of the lens. A homogeneous cooling of the molten plastic is the main task for the cooling channel layout. For complex geometric shapes, with e.g. big differences in wall thickness, this is a very difficult task. Systematising the design process with a reproducible tool design quality and achieving an independence from the designer are the two main goals for many publications in recent years dealing with the automatic cooling channel design in injection moulding.

\section{Automated cooling channel design in current research}

So far, there are only a few automatic methods for the thermal mould design. Due to the increasing functional density and more and more complex part geometries, the development of a temperature system needs to consider various boundary conditions and restrictions. Therefore, the development of a computer-aided optimisation strategy to automatically determine an optimal cooling channel design for a given part, is still subject of ongoing research. In the following, some existing approaches in current research are presented.

$L i$ and $W u$ propose an evolutionary approach with ad hoc operators to concurrently optimize the topological connection and geometric position of a cooling system. A mixed encoding scheme is developed to translate a suitable solution, which is represented in the form of a variant-length chromosome. Ad hoc evolutionary operators and parameters adapting to the characteristics of cooling system design are devised [8]. All the segments of the generated cooling channels by this approach are designed along the $\mathrm{x}, \mathrm{y}, \mathrm{z}$ direction, so the 
distribution of the cooling channel is relatively simple. It is difficult to design cooling channels that can meet complex cooling requirements e.g. for parts with a freeform surface.

Given a model of a plastic part to be fabricated by rapid tooling, Wang, Yu and Zhang present an automatic method for designing conformal cooling circuits. A Voronoi Diagram (VD) on the plane is defined by a collection of $\mathrm{n}$ sites, by which the diagram divides the plane into $\mathrm{n}$ sub-regions such that the closest site of all the points within a sub-region $\mathrm{S}_{\mathrm{i}}$ is the site $\mathrm{S}_{\mathrm{i}}$ inside this region. A Centroidal Voronoi Diagram (CVD) is a VD where each Voronoi site is also the mass-centre (centroid) of its Voronoi region. The separated offset surface of the part will be served as the conformal surface. The conformal surface will be discretely refined by CVD. Afterwards the resulting conformal cooling circuit, which is along the Voronoi sites, will be determined. The approach is advantageous regarding automatic generation and producing the cooling channels conformal to the shape of products [9]. The generated cooling circuits are very dense and follow the shape of the part, which greatly reduces the cooling time. Unfortunately, this method does not consider the heat transfer properties of the part, and the form of the study case, which is a hemispherical helmet, is relatively simple. If this method is applied to a part that has a complex geometry, there is no guarantee to reduce part warpage.

Zhang et al. propose a design process where they create equidistant lines to the part surface by an automatic algorithm. They specify the diameter, the offset to the part and the distance between two channel sections. Then the script based on $\mathrm{C}++$ creates spiral-like cooling channels with a constant distance to the part automatically. This is a purely geometric approach to the conformal cooling design process not taking into account the increased cooling demand for part areas such as ribs, changes in wall thicknesses etc. [10].

Jahan et al. develop a more elaborate approach and propose a thermo-mechanical design optimisation of cooling channels and the injection mould. The cooling channels cannot withstand mechanical forces, so that channels close to the cavity create high stresses in the remaining material. This leads to the conclusion that not every cooling channel design that would create an optimal thermal result for the part is suitable for production. Therefore, further factors as the created stress in the mould material needs to be considered. The geometry is parameterized in a similar way as in the aforementioned publication. The diameter and the pitch distance between the centreline of the cooling channel and the simple cup-shaped part are varied. By a design of experiments (DoE) several cooling channel designs are tested and the best design is chosen by the criteria minimum yield stress and minimum cooling time. In conclusion, the outcome of this study heavily depends on the weighting of the input parameters of the user, which does not assure that a global optimum can be found. Also, the resulting part warpage is not considered but only the achievable cooling time. [11]
Several authors have taken the cooling demand of the part into the focus of their research. They do not only consider the geometry of the part but also the thermal state inside the mould. By defining a quality function that describes the homogeneity of the cooling, they develop a criterion for the part quality at the end of the cooling phase such that the thermal problem can be solved by an optimisation software.

Rhee et al. want to combine the classical drilling of cooling channels with the advantages of a thermal optimisation. They parameterise their geometries by using baffles to achieve high cooling performance close to the cavity. They calculate density and offset of the baffles and use bumper from the automotive sector as a demonstrator. Based on a quality function that sums up the differences of the surface temperature to a pre-set demoulding temperature, the cooling channels are developed automatically by the software in an iterative optimisation process. The software varies the distance and the length of the baffles to calculate an optimal heat transfer based on a similar quality function as Agazzi et al.. With the developed methodology a cooling circuit for large moulds can be generated. However, highly curved component areas could not be optimally cooled and exhibit large temperature differences between the upper and lower component sides. This is also because the baffle tubes cannot be manufactured uniformly in these areas [12].

Summing up the current work in research, a lot of different approaches have been proposed to design cooling channels for injection moulds. Most of the times, a start design for the cooling channels is given or the degrees of freedom are limited to restrict calculation time. The designer is needed to choose between the different options and set up the optimisation space. In order to solve the above problems, an approach that uses the inverse cooling channel design will be developed. It can automatically determine complex cooling channel designs to meet the cooling requirements of the part.

\section{Calculation of an automated cooling channel design}

The IKV has developed a methodology to calculate the optimal temperature distribution inside the mould. The aim of this methodology is to calculate a thermal regime inside the mould such that the part properties are as homogeneous as possible after the cooling phase. The methodology is divided into two steps. At first, an optimal thermal regime is calculated based on a quality function and the local cooling demand of the part. In the second step, a cooling channel design can be derived based on the results of the first step. During the first step, the cooling phase of the injection moulding process is calculated and optimized. This methodology has originally been developed and patented by Agazzi et al. and has been further developed by Nikoleizig et al. [13-18]. As for every optimisation, the software needs a quality evaluation of the part. Therefore, a function has been developed that sums up the differences in 
temperature and density in the part. The thermal regime is calculated only in a surrounding mould envelope close to the part $(\Delta \mathrm{x}$ of $10 \mathrm{~mm})$. The temperature distribution inside the mould close to the part defines the thermal gradients and therefore the thermal fluxes from the part inside the mould.

In this methodology, the assumption is made that a homogeneous distribution of temperature and density leads to a part with minimal warpage. The quality function has been defined as follows [18]:

$$
\begin{aligned}
Q\left(T_{K K 0}\right)= & \sum_{i=1}^{m} \int_{0}^{t_{\text {cool }}}\left(\frac{T_{\text {ejec }}-T_{F}\left(x_{i}, t ; T_{K K 0}\right)}{\omega_{i}}\right)^{2} d t \\
& +\sum_{j=1}^{k} \int_{0}^{t_{\text {cool }}}\left(\frac{\overline{\rho_{F}}-\rho_{F}\left(x_{j}, t ; T_{K K 0}\right)}{\omega_{j}}\right) d t
\end{aligned}
$$

The temperatures $T_{K K 01} ; \ldots ; T_{K K 0 n}$ are searched for on the mould envelope $\Gamma_{W}$ surrounding the moulded part, which minimises the error square sum of the quality function for all $T_{K K 0 i} \in[a, b]^{n}$ for $0<t<t_{\text {cool }}$.

The objective function consists of two terms, which determine on the one hand the efficiency, i.e. the speed of solidification, and on the other hand the quality, i.e. the homogeneity, of solidification. In the first term, the local differences between the desired demoulding temperature $T_{E j e c}$ and the local moulding temperatures $T_{F}$ is evaluated over the part surface $\Gamma_{F o}$ and the cycle time. In the second term, the difference between the averaged density $\overline{\rho_{F}}$ and the local density $\rho_{F}$ is evaluated over the area inside the part $\Gamma_{F i}$ and the cycle time. Weighting of the two terms is possible via $\omega_{i j j}$. The weighting factor is a multiplicative factor which makes sure the corresponding term converges quicker than the other. Only the relative size to each other is important. Based on previous research, the constant values $\omega_{i}=50^{\circ} \mathrm{C}$ and $\omega_{j}=100\left(\frac{\mathrm{kg}}{\mathrm{m}^{3}}\right)$ are chosen for all tests in this publication. Based on a realistic starting temperature distribution, that takes into account the cooling of the melt during the filling process, the cooling phase is calculated. Then, the temperature distribution on $\Gamma_{W}$ is iterated via a conjugated gradients method for high number of degrees of freedom. Finally, the calculated optimized thermal regime is analysed and iso-surfaces are generated by the following equation:

$0=\int_{0}^{t_{c o o l}}\left(h_{M \rightarrow C C}\left(T_{\text {loc }}-T_{\text {coolant }}\right)-\left|\lambda_{M} \operatorname{grad} T_{\text {loc }}\right|\right) d t$

This equation expresses the equilibrium between the locally existing absolute heat flux $\left(\left|\lambda_{m} \operatorname{grad} T_{l o c}\right|\right)$ and a theoretical heat flux inside the mould steel created by a cooling channel at this specific position with a coolant temperature detailed in the datasheet of the used plastic material. The heat transfer coefficient from the mould into the cooling channel is given by $h_{M \rightarrow C C}$. By this method "iso-flux" surface are derived that indicate the position of the cooling channels.

\section{Investigation on the influence of the quality} area on the optimisation quality

So far the proposed methodology has only been used for thinwalled injection moulded parts $[13,19,20]$. The term "standard wall thickness" will be used in this publication for parts with wall thicknesses below $3 \mathrm{~mm}$. Up to this value, the cycle time in the injection moulding process is usually lower than $30 \mathrm{~s}$. This means, that there are high cooling rates and the temperature gradients over the wall thickness are comparably low. In comparison, the wall thickness of optics for applications such as headlights or indicators in a car is often considerably thicker. For these types of parts, wall thicknesses may vary between 8 and $25 \mathrm{~mm}$. In the latter case, the cycle time can be up to twenty minutes and the temperature gradients are comparably high, because the surface temperature of the part will quickly approach the cavity wall temperature, whereas the core of the part stays molten for much longer time. Due to the low thermal conductivity of the plastics, these gradients are only slowly released. Different areas of the part need to ensure different optical tasks. Therefore, big changes in wall thickness are the current state of the art.

\section{Differences between the chosen geometries}

In this study, three different geometries are investigated. A standard injection moulded part with low wall thicknesses and two optical parts: a simple biconvex lens geometry and a complex automotive lens system used in blinker lights. In contrast to standard injection moulding processes, thickwalled optics require a different set of process parameters. A slow filling speed is chosen such that low shearing and thus a low orientation of the molecular chains can be achieved. A high orientation of the molecules lead to a low optical quality due to birefringence [21]. To further decrease the load on the melt, a high mould temperature or even a variothermal process is chosen. These process parameters usually lead to high shrinkage of the plastic material, which can be compensated by a closing movement of the movable side of the mould to ensure constant contact with the polished cavity surface. This processing variant is well known as injection compression moulding [22]. For the following study, the injection compression moulding is considered in a way that a constant heat flux from the plastic melt into the mould is assumed.

\section{Adaption of the methodology}

In the existing simulation process an area is defined for the temperature and the density evaluation. This quality area is placed within the part and has a constant distance to all part surfaces. For the inner properties of thick-walled polymer parts the cooling of the inner material is critical, especially for optical parts. The present methodology with a quality area 
on the surface of the part geometry does not consider the cooling of the inner part (2D approach). Therefore, the quality area is adapted for thick-walled polymer parts. In the first adaption, the whole volume within the quality area of the current approach is taken into account during the optimisation calculation (3D approach) with the aim of taking into account the entire inner area of the component. In the second adaptation, a new surface is calculated in the geometrical centre (mid-plane approach) and used as quality area for Eq. 3. The approach for optical thick-walled parts made of amorphous thermoplastic follows the idea to achieve the glass transition temperature at any point of the component regardless of the local thickness at the same process time. For semicrystalline thermoplastics, the goal is to reach simultaneously the recommended demoulding temperature. This approach is shown exemplarily in Fig. 1.

The centre surface for the lens is designed by using CADsoftware. For more complex geometries, the centre surface is calculated using a python script. The upper and lower surface of the component is extracted as a cloud of points and for every point with the same $\mathrm{x}$ - and $\mathrm{y}$-value the difference in $\mathrm{z}$ direction is calculated. Thus, a point of clouds in between the surfaces is calculated, which is used to create the centre surface independent of the complexity of the part surface. The quality function introduced by Nikoleizig et al. is used to investigate the thermal homogeneity of the part at the end of cooling (see Eq. 1). In this paper, the two new approaches described in Fig. 1 are investigated and compared to the current 2D approach.

For efficiency reasons, the thermal and the density term within the quality function needs to be adjusted. The integral over the quality area $\Gamma$ (changing for the three approaches) allows a comparison of the quality function for all three methodologies and is only calculated for the last time step, reducing the calculation time significantly.

$$
\begin{aligned}
Q\left(T_{K K 0}\right)_{O p t}= & \int^{\Gamma}\left(\frac{T_{e j e c}-T_{F}\left(x_{i}, t ; T_{K K 0}\right)}{\omega_{i}}\right)^{2} d \Gamma \\
& +\int^{\Gamma}\left(\frac{\overline{\rho_{F}}-\rho_{F}\left(x_{j}, t ; T_{K K 0}\right)}{\omega_{j}}\right) d \Gamma
\end{aligned}
$$

Fig. 1 Different approaches for the quality area

$$
\text { the quality area }
$$

Using the integral over the quality area $\Gamma$ takes the size of the elements of the simulation into account. To allow a comparison the quality function is calculated for all three methodologies only for the last time step, reducing the calculation time significantly.

The two terms are independent from each other. The first term describes the difference in temperature and in the current methodology it is evaluated over the part surface. On the part surface, the influence of temperature differences in the mould is the highest. Furthermore, this term describes the efficiency of the process, as soon as the part surfaces is solidified, the part can be demoulded. The 3D approach should therefore take into account a more homogeneous cooling over the part thickness. The second term describes the local homogeneity of the density and is a measure for the warpage potential of the part. An inhomogeneous density will lead to an unwanted bending of the part. The density is evaluated on a surface in the part where the temperature influence of the mould is smaller and the pressure differences have a larger effect [18]. In the following, the influence on the optimisation result of these quality areas is investigated. Three parts that cover a wide range of plastic products are chosen.

\section{Testing procedure}

\section{Specimen}

The technical part (box part) has a size of $80 \cdot 140 \cdot 40 \mathrm{~mm} 3$ and an approximate shot volume of about $68 \mathrm{~cm}^{3}$. The demoulding incline is $1^{\circ}$ for the ribs and box section and $0.67^{\circ}$ for the cold runner (cf. Figure 2). The wall thickness at the base plate is $2 \mathrm{~mm}$ and at the highest point of the ribs is $1 \mathrm{~mm}$.

The simple optical lens geometry is a biconvex lens. Due to the low refractive index of the considered plastic material, the part is considerably thick with a maximum wall thickness of $10.21 \mathrm{~mm}$. The part is visualised in Fig. 3. It is usually made of amorphous plastics such as PMMA or PC. The diameter of the optical section is $50 \mathrm{~mm}$ and the overall diameter is $70 \mathrm{~mm}$. The approximate shot volume is $40 \mathrm{~cm}^{3}$. [mm].

The last part is related to an automotive part being used as light guide for the indicator lights. In contrast to the biconvex lens, this part has a lot of changes in wall thickness, from a

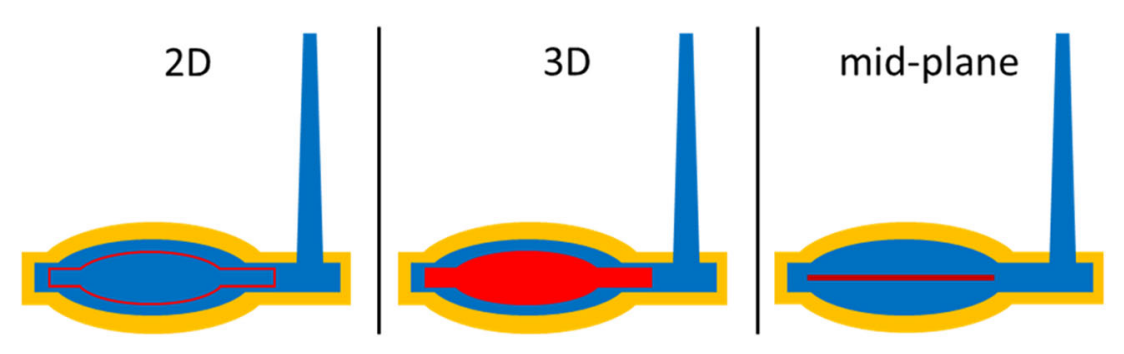


Fig. 2 Dimension of the box part

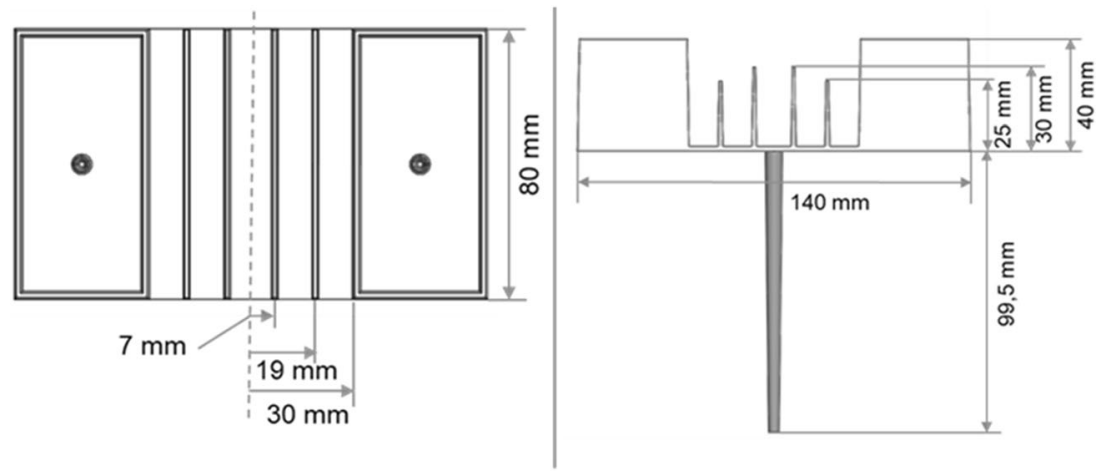

minimum of $2.5 \mathrm{~mm}$ up to a max. Thickness of $15.26 \mathrm{~mm}$. This leads to the very specific challenge of homogeneous cooling. The thin parts cool down very quickly due to the close contact to the cavity wall, whereas the low conductivity of the plastic leads to an hot molten core and it is difficult to evacuate the heat. The shot volume is $35 \mathrm{~cm}^{3}$. In Fig. 4, the overall dimensions are shown.

\section{Process conditions \& material}

The box part is produced with a semi-crystalline Polybutylene terephthalate (PBT) from the type Ultradur B4520, BASF SE, Ludwigshafen, Germany, whereas for the optical parts an amorphous polymethyl methacrylate (PMMA) 7 N Roehm $\mathrm{GmbH}$, Weiterstadt, Germany is used. In the following thermal optimisations, only the cooling phase is optimised. As a starting condition, the temperature distribution at the end of filling is used. This temperature distribution is calculated using the injection moulding software Sigmasoft V5.3, Sigma Engineering GmbH, Aachen. The temperature distribution is then mapped onto the geometry and transferred into the Comsol software environment. The process parameters are shown in Table 1. The calculation of the cooling phase is iterated 50 times and the thermal boundary conditions are

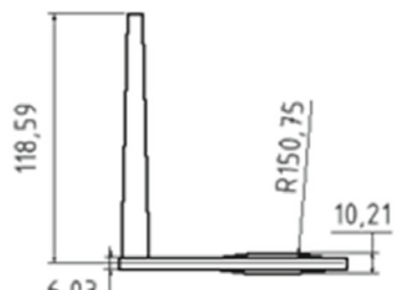

6,03

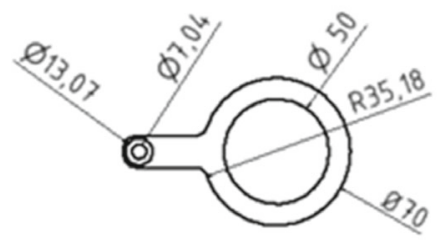

Fig. 3 Biconvex lens geometry
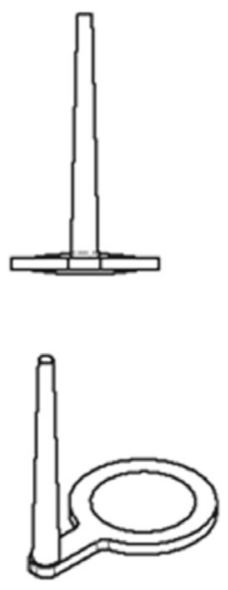

[mm] adapted after each iteration. At the end of the optimisation, the convergence of the quality function is verified.

\section{Results of thermal optimisation}

After finalisation of the thermal optimisation in the Comsol software environment, the iso-flux surfaces indicating the location of the cooling channels can be displayed and transferred to a CAD software. Therefore, the iso-flux surfaces are selected according to the coolant temperature of $80^{\circ} \mathrm{C}$. The cooling channels are constructed along the respective isoflux surface. Any geometric shape for the creation of the cooling channels is conceivable, but in this paper, all cooling channel cross-sections are created around the iso-flux surface to enable a comparison. Figure 5 shows the design process using the example of the indicator lens.

\section{Optimisation of the temperature distribution with Comsol}

After the thermal optimisation within the multiphysics software Comsol, the temperature distribution on the surrounding mould contour is available, offering the best possible temperature distribution to the corresponding quality area. Since the optical performance is not only affected by a certain area but by the whole component that is penetrated by the light, the temperature distribution over the whole geometry is evaluated to analyse the simulation results.

The temperature distribution of the box part at the end of cooling shows high differences due to the process settings. In the short cycle time, the inner core of the part is still at a quite high temperature at the end of cooling. In addition, the temperature of the cold runner is taken into account, which still has a very high temperature due to its high wall thickness. It can be seen, that the $3 \mathrm{D}$ approach lowers the overall average temperature because the quality area is much larger. The overall part temperature for the $2 \mathrm{D}$ approach is quite high because only the part surface is optimised to $110{ }^{\circ} \mathrm{C}$. The aim of the optimiser is to get the whole part close to ejection temperature. These results are summarised graphically in Fig. 6. 
Fig. 4 Automotive optical part (indicator)

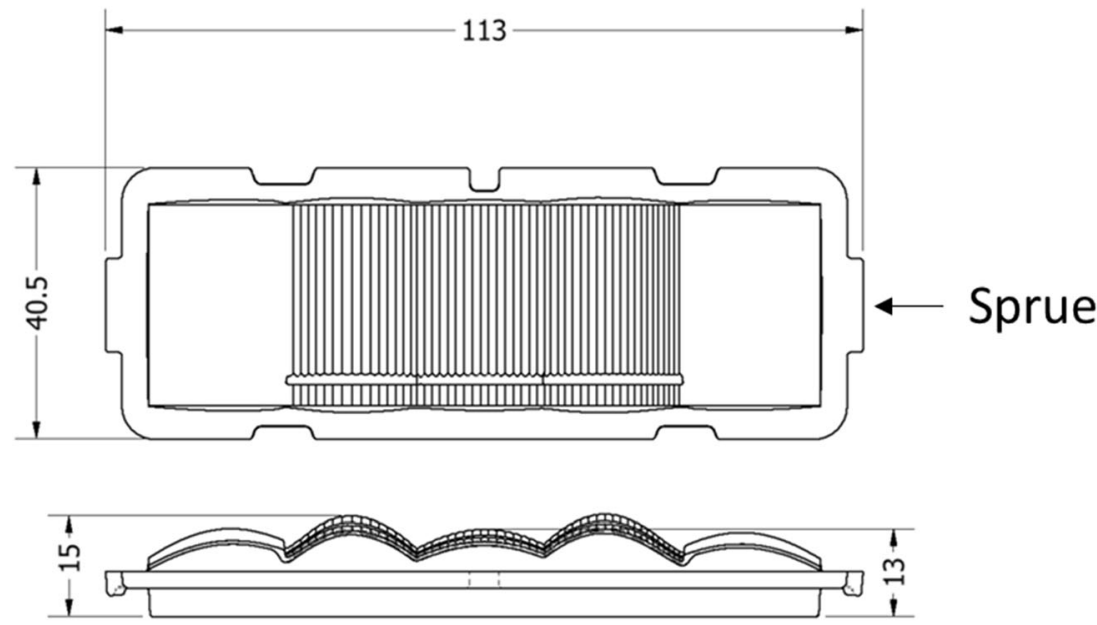

Figure 7 shows the temperature distribution within the biconvex lens for the three different quality areas. The $2 \mathrm{D}$ surface as quality area leads to a significantly warmer lens centre. In contrast, the 3D surface takes the temperatures of the lens centre in the quality function into account and the result is a much cooler lens. The very strong cooling in the centre of the lens becomes clear in the mid surface optimisation. In this case, a very strong cooling of the outer peripheral regions is also noticeable. Comparing the average temperature value and the corresponding standard deviation, it becomes clear that the mid-plane approach leads to a significantly lower lens temperature, but the standard deviation remains the same and therefore a more homogeneous cooling over the entire component height is not achieved. The 3D-approach generates a less significant cooling of the lens, but the standard deviation is reduced. This suggests a more homogeneous temperature distribution.

In the case of the indicator lens, the thick-walled area inside the lens is significantly warmer than the glass transition temperature $\mathrm{T}_{\mathrm{G}}$ of the material for the $2 \mathrm{D}$ approach (Fig. 8). Concerning the $3 \mathrm{D}$ approach, the area of the mould envelope seems to heat the component rather than cool it down. Finally, the mid-plane shows a clear alternation between warm and cold areas, which is related to the thick and thin-walled areas of the indicator lens. The average temperature, shown in Fig. 9, increase slightly in the 3D surface compared to the 2D surface and shows a slightly higher standard deviation. The results of the optimisation over the mid-plane show, as already for the biconvex lens, the lowest average temperature, but in this case, the standard deviation is only slightly below the one of the 2D surface.

In Fig. 9, the results are summarised. The three different approaches lead to varying temperature distributions inside the different parts indicating different results for the part warpage as the final target value. Therefore, cooling channels are derived based on the procedure mentioned in the following chapter. The error bars indicate the standard deviation of the temperature distribution over the respective part. It can be seen that the box part has considerably high standard deviations, which is due to the small wall thickness and short cycle times, which cool down quickly the outside to a solid demoulding temperature, but the inside is still warm.

\section{Derivation of cooling channels based on thermal optimisation}

The process of deriving cooling channels is a geometrical construction work, which is currently done manually. In future work, this step is going to be automated. The iso-flux surfaces are taken from each thermal optimisation and used as an indication for the location of the cooling channels. The cooling channels are designed following the design rules of additive manufacturing and the mechanical stability of the mould is ensured by keeping at least one diameter distance to the mould cavity. Nine designs have been developed based on the three representative part geometries and the three respective evaluation methods. It has been focused on avoiding dead ends with stagnating cooling fluid and
Table 1 Process parameters for the three parts

\begin{tabular}{lllll}
\hline Moulding Parameter & unit & Box part & Biconvex lens & indicator \\
\hline Cooling time & {$[\mathrm{s}]$} & 10 & 240 & 675 \\
Starting temperature (depending on flow length) & {$\left[{ }^{\circ} \mathrm{C}\right]$} & $168-281$ & $140-240$ & $155-240$ \\
Mould temperature & {$\left[{ }^{\circ} \mathrm{C}\right]$} & $88-165$ & $82-94$ & $104-250$ \\
Ejection temperature & {$\left[{ }^{\circ} \mathrm{C}\right]$} & 110 & 110 & 110 \\
\hline
\end{tabular}


Fig. 5 Schematic process flow for the construction of the cooling channels

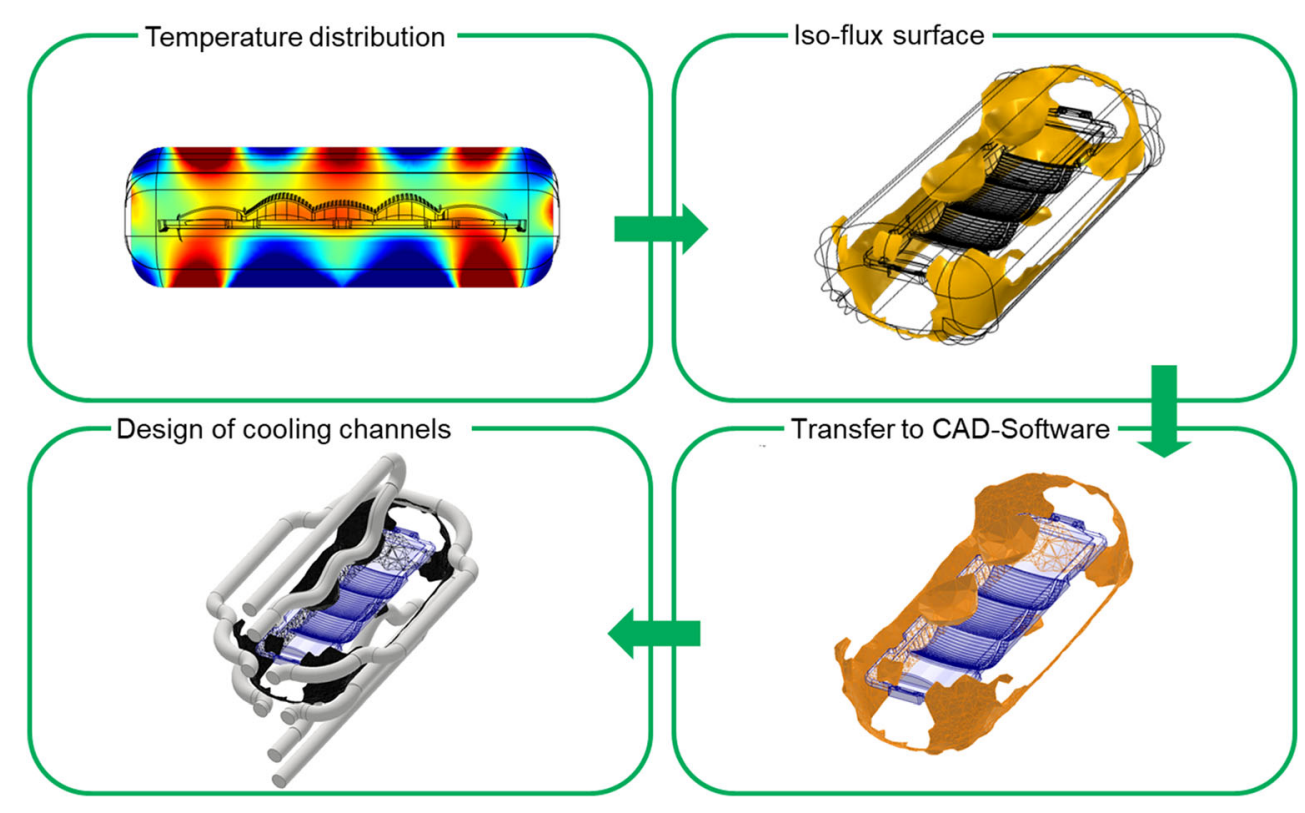

big radii have been chosen. The derived cooling channel designs can be seen in Fig. 10.

The cooling channels for the box part have a diameter of $8 \mathrm{~mm}$ in the outer contour to minimise the flow resistance and between the ribs section the diameter is lowered to $3 \mathrm{~mm}$ due to the geometrical constraints. The diameter for the lens geometry is chosen as constant $8 \mathrm{~mm}$, which suits best the resulting surfaces. The main difference between the three derived cooling channels is the distance to the geometry. The cooling channels of the indicator geometry have a constant diameter of $12 \mathrm{~mm}$ enabling a high water flow and thus a high heat dissipation.

\section{Simulative validation of the derived cooling channel design}

Finally, the derived cooling channel designs need to be validated by a process simulation. The process simulation software Sigmasoft is chosen to investigate the development of the part warpage. The injection moulding process is simulated and an analysis of the part warpage is performed.

\section{Setup of the simulation}

The same materials and process parameters regarding cooling time, melt/mould temperatures are chosen (cf. Table 1). The filling time of the respective parts has been set to suitable values. The box part is filled quickly to ensure that no melt solidifies before the end of filling. In contrast, the optical parts are filled considerably slow to minimise shear stress that reduces the optical quality. The volume flow for the box part is set to $50 \mathrm{~cm}^{3} / \mathrm{s}$, whereas the lens geometry and the indicator are filled with a constant low volume flow of $10 \mathrm{~cm}^{3} / \mathrm{s}$ (lens) and $20 \mathrm{~cm}^{3} / \mathrm{s}$ (indicator). For the box part, a total of 15 heating cycles is chosen to simulate the periodical heating of the injection mould. Temperature distribution and part warpage are evaluated at the 16 th cycle. Due to the long cycle time of the
Fig. 6 Temperature distribution for the box part in the mid-section

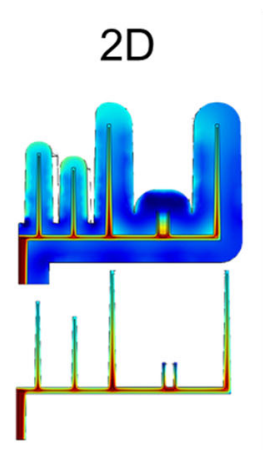

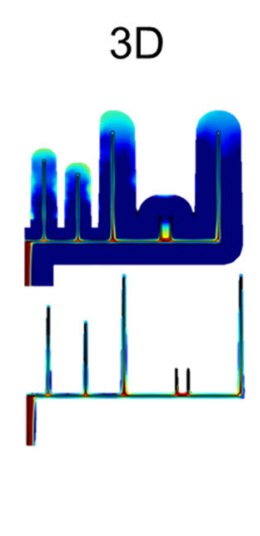

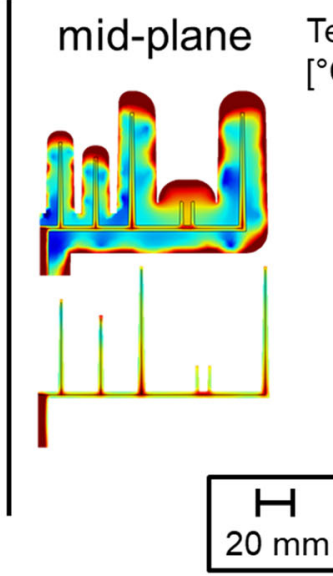

Temperature $\left[{ }^{\circ} \mathrm{C}\right]$ 180 
Fig. 7 Temperature distribution over the midsection of the biconvex lens after optimisation
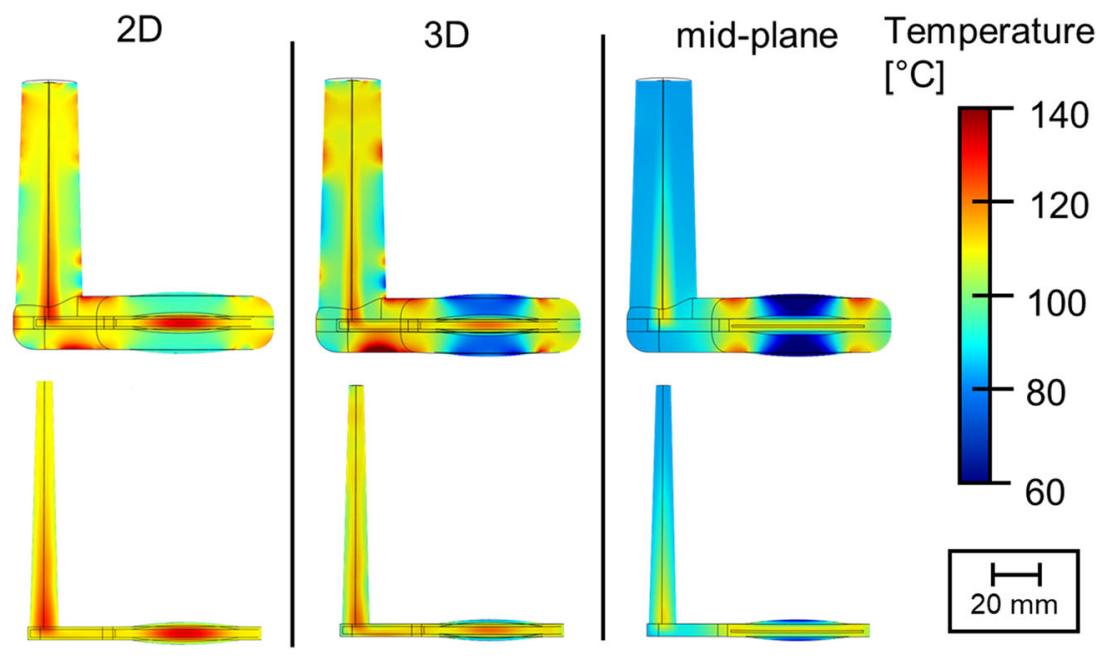

optical parts, five heating cycles are chosen and the evaluation is performed at the 6th part. As material data, the standard material cards from the Sigmasoft database are used.

\section{Validation simulation of derived cooling channel designs}

The simulations have been analysed in regard of two parameters. First, a homogenous temperature was required by the quality function (cf. Eq. 3). The averaged temperature and density distribution were taken out of the simulation at the point of ejection. These results are summarised in the following Fig. 11:

The calculated temperature distribution of the injection moulding simulation with the respective cooling channel design shows that for the box part, the mid-plane approach as a quality area leads to the lowest average temperature. However, this is only $2{ }^{\circ} \mathrm{C}$ lower than with the $2 \mathrm{D}$ approach. The $3 \mathrm{D}$ approach achieves the highest average temperature with the lowest standard deviation. The measured displacement of the box part at the three marked positions is lowest with the 2D optimisation approach. In spite of a low average temperature and the same standard deviation, the displacement is greatest in the case of the mid-plane optimisation.
In the case of the biconvex lens, the simulation result with the mid-plane approach as quality area shows the lowest average temperature, but also a slightly higher standard deviation. The average temperature is in all three cases lower than the glass transition temperature of $110^{\circ} \mathrm{C}$ and they are within a range of $2{ }^{\circ} \mathrm{C}$. Therefore, the approach should be chosen which achieves the most homogeneous cooling to minimize stress and thus the approach with the smallest standard deviation. Considerably lower standard deviations and thereby more homogeneous temperature distribution are shown by the $2 \mathrm{D}$ and $3 \mathrm{D}$ approach. The significantly lower standard deviations of the lenses compared to the box part are due to the longer cooling times required for thick-walled optical components. The 2D approach leads to a slightly lower average temperature. The calculated displacements at the indicated points show no significant difference. The results of the indicator lens show the lowest average temperature within the part over all investigated quality areas. The average temperature is lowest in the 3D approach, but the results of all three approaches are quite near to each other due to the long cooling time. The mid-plane approach has the lowest standard deviation of the mean temperature but this is not significantly. As with the biconvex lens, the differences in displacement at the measurement points of the indicator lens are not significant.
Fig. 8 Temperature distribution over the midsection of the indicator lens

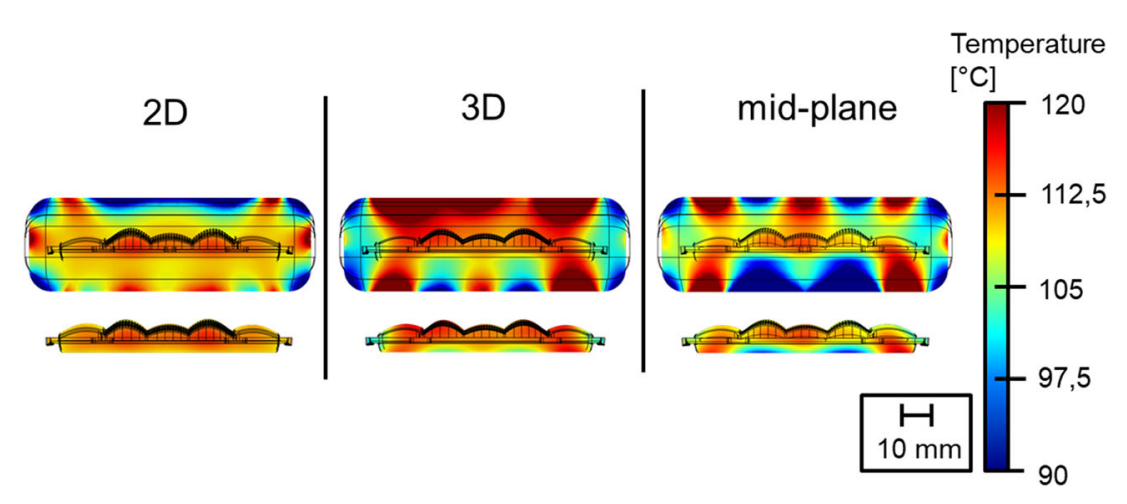


Fig. 9 Average Temperature of the test specimen after optimisation

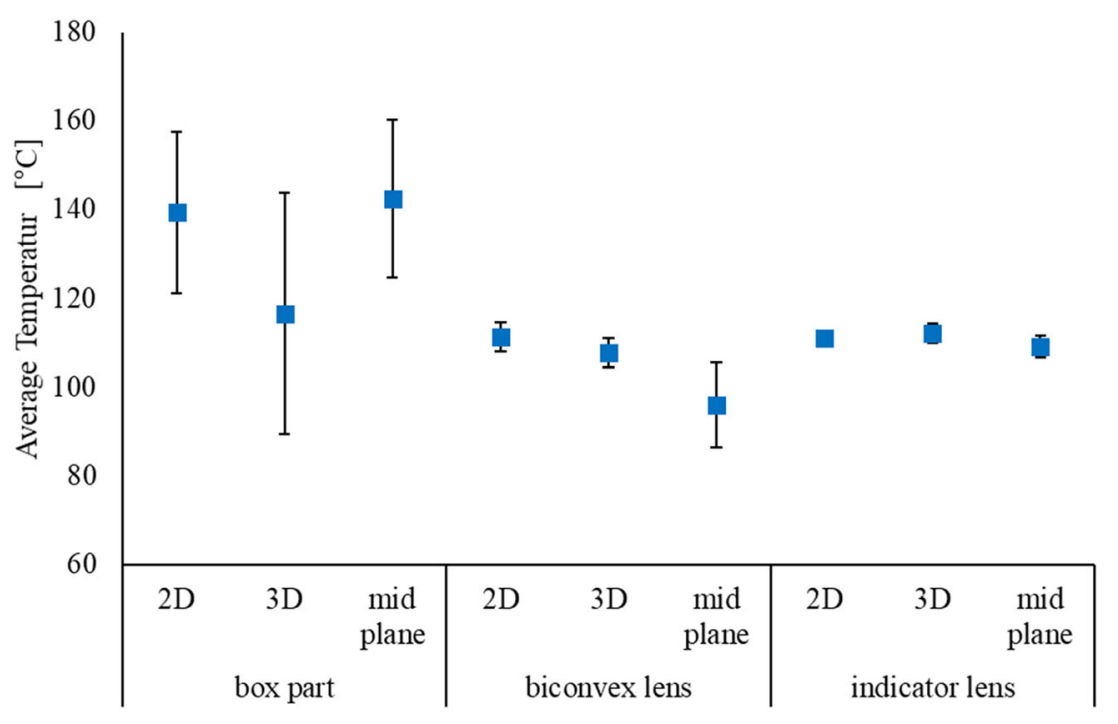

There is no significant difference between the results of the density of both simulation software. The simulation of the lens geometries shows that in the validation in Sigmasoft with the generated cooling channels a more heterogeneous density distribution is created in the components than calculated in the optimization. While the average density of Sigmasoft is calculated slightly lower for the semi-crystalline material, this is slightly higher for the amorphous thermoplastic than the values from the optimization software Comsol.

There is a difference of 5 to $20^{\circ} \mathrm{C}$ between the simulation results of Sigmasoft and the calculated temperatures of Comsol. The results of Comsol show that the 3D approach is best suited for the biconvex lens and the box part and for the indicator lens the mid-plane approach. While the thermal optimisation aims to fulfil the criteria of the quality function at a predefined point in time, the injection moulding simulation calculates the cooling with the created cooling channels without a given temperature and a constant cooling of the cooling channels. These results show that a shorter process time is theoretically possible with the current conformal cooling channel design.

The final part warpage is analysed for three representative points for each part. The box part is analysed exemplarily along the symmetric mid-plane through the part. The other measurement points on the part give the same indications. Similar points have been chosen for the biconvex lens and the indicator light. The results are summarised in Table 2.

\section{Conclusion \& Outlook}

In this publication, a method has been presented to objectively evaluate the thermal mould design for injection moulds. Based on an existing approach the quality area for the evaluation of the quality function has been changed to understand the influence of this factor. It could be shown, that the thermal optimisation of three presented parts shows very different results depending on the chosen quality area. The resulting
Fig. 10 Different cooling channel designs for the three approaches

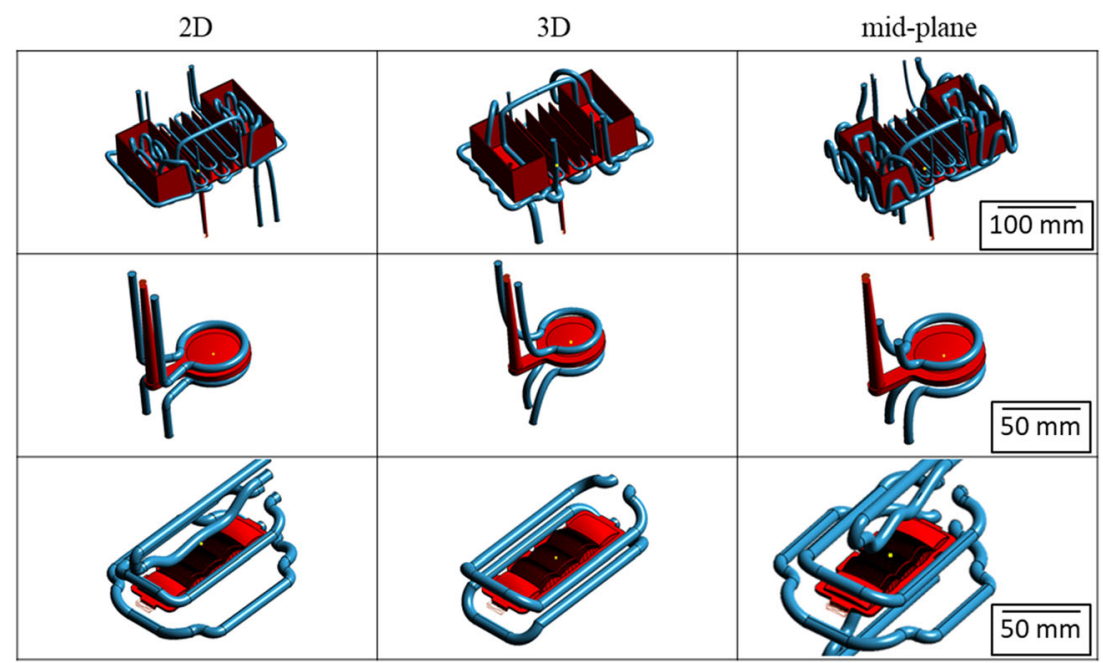


Fig. 11 Resulting average temperature in the three parts

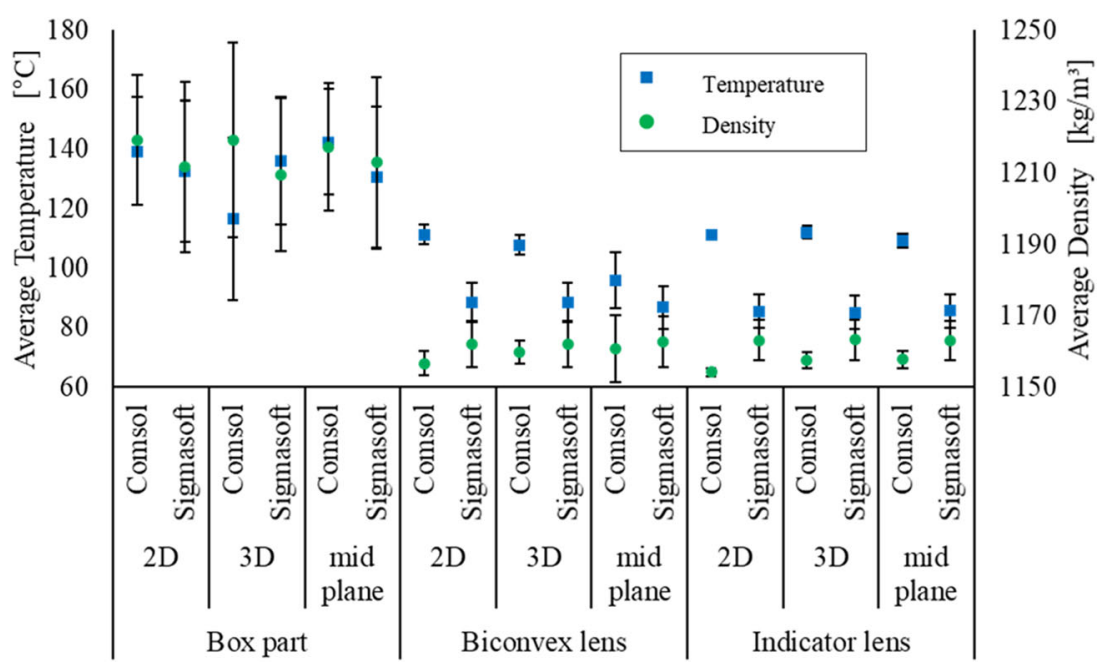

temperature distribution in the part and the mould differ significantly. The derived cooling channels designs are not very different for the biconvex lens but differ significantly for the box part and the indicator lens. The different cooling channel design do not result in a significant difference in temperature distribution at the end of the cooling calculation in Sigmasoft. Nevertheless, the resulting part warpage shows comparable differences for the box part, where the 2D approach delivers the best results. The approach that for thin-walled parts the homogenisation of the surface temperature is sufficient, can be continued.

For thick-walled geometries, it has been shown that a volumetric approach for the quality area of Eq. 3 results in better temperature distributions at the optimization calculation. However, the geometry of the component has an influence on which volumetric approach is preferable. For very simple thick-walled components, the mid plane approach provides the lowest average temperature; for more

Table 2 Resulting part warpage for the different approaches.
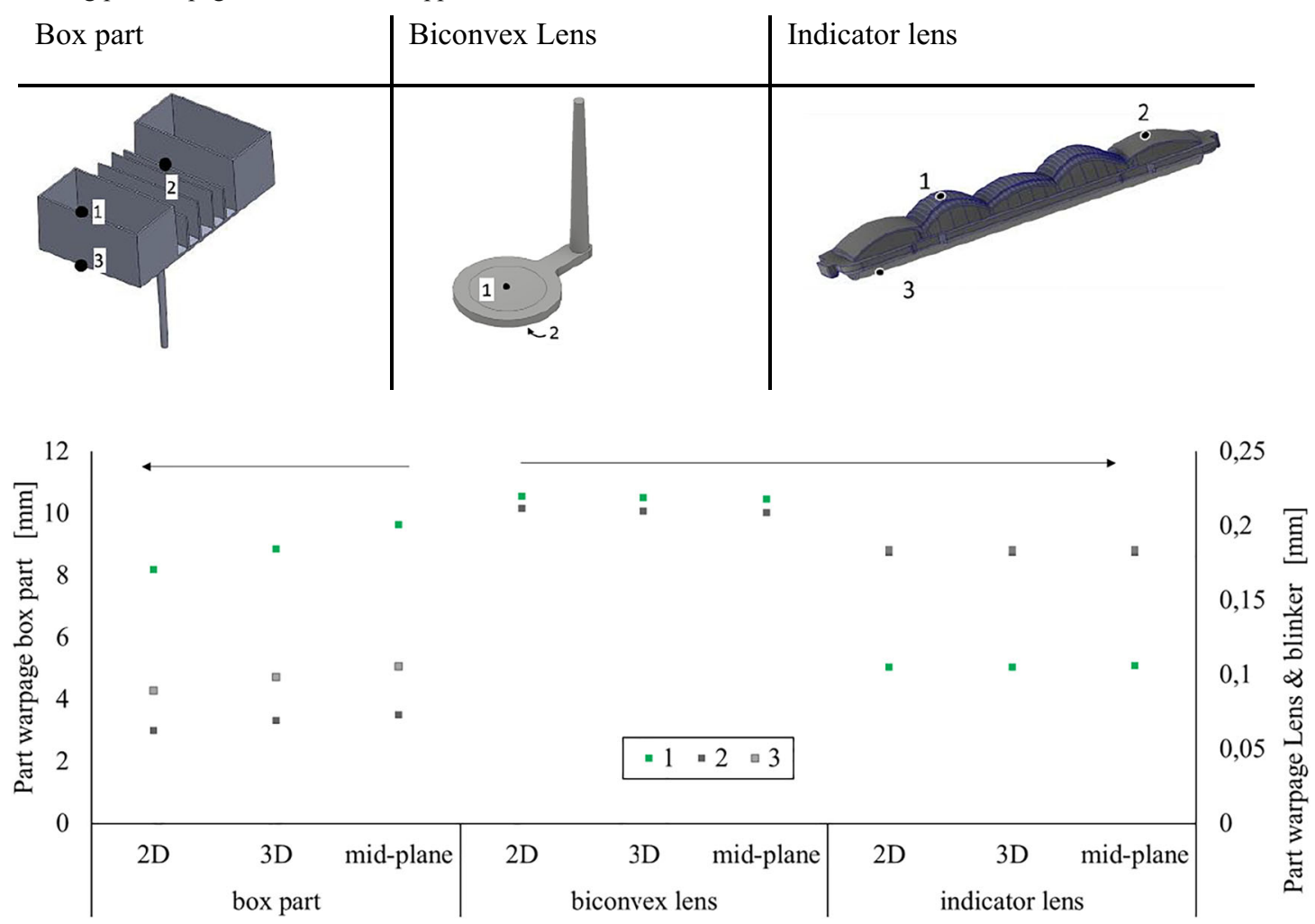
complex thick-walled components with wall thickness jumps, the volumetric approach provides the best results as the basis for calculation. Based on the results of this paper, future parts will be analysed with regard to the quality area to choose before starting with the thermal optimisation process. Based on a classification of the parts (thick-walled, many wall thickness jumps, etc.), the correct approach for the quality area will be chosen.

In future work, the formulation of the quality function will be investigated. The aim is to ensure that both terms have the same order of magnitude and can be used without adaption to any geometry. Whether this normalisation of the quality function and a change of the weighting factors $\omega_{1 / 2}$ does further influence the results of this methodology, is the goal of the current ongoing research. Furthermore, more complex material models such as a cooling-rate dependent pvT-model will be implemented into this optimisation and investigated.

In the case of the thick-walled optics, the process of compression moulding, as an extension of the current methodology, will be implemented as this specific process is vital for high quality optical lenses and current state of the art in optical manufacturing.

Acknowledgments The presented investigations were carried out at RWTH Aachen University within the framework of the Collaborative Research Centre SFB1120-236616214 "Bauteilpräzision durch Beherrschung von Schmelze und Erstarrung in Produktionsprozessen" and funded by the Deutsche Forschungsgemeinschaft e.V. (DFG, German Research Foundation). The sponsorship and support is gratefully acknowledged.

Funding Open Access funding enabled and organized by Projekt DEAL.

\section{Compliance with ethical standards}

Conflict of interest The authors declare that they have no conflict of interest.

Open Access This article is licensed under a Creative Commons Attribution 4.0 International License, which permits use, sharing, adaptation, distribution and reproduction in any medium or format, as long as you give appropriate credit to the original author(s) and the source, provide a link to the Creative Commons licence, and indicate if changes were made. The images or other third party material in this article are included in the article's Creative Commons licence, unless indicated otherwise in a credit line to the material. If material is not included in the article's Creative Commons licence and your intended use is not permitted by statutory regulation or exceeds the permitted use, you will need to obtain permission directly from the copyright holder. To view a copy of this licence, visit http://creativecommons.org/licenses/by/4.0/.

\section{References}

1. Pötsch G, Michaeli W (2008) Injection molding. An introduction. Hanser, Munich

2. Hopmann C, Menges G, Michaeli W, Mohren P (2018) Spritzgießwerkzeuge. Auslegung, Bau, Anwendung. Carl Hanser Verlag, Munich

3. Malloy RA (2011) Plastic part design for injection molding. An introduction. Hanser, Cincinnati, Ohio

4. Frekers V (2019) Improved part properties via virtual DoE. SIGMA Engineering GmbH. https://www.sigmasoft.de/en/press/ pressreleases/improved-part-properties-via-virtual-doe/. Accessed 06 March 2020

5. NN (2017) Process Optimization analysis (Concept). https:// knowledge.autodesk.com/support/moldflow-insight/learn-explore/ caas/CloudHelp/cloudhelp/2018/ENU/MoldflowInsight/files/ GUID-5EC36B17-F7B4-4083-8872-E86AA04BA849-htm.html. Accessed 06 March 2020

6. Lin CM, Wang CK (2013) Processing optimization of optical Lens in the injection molding. Adv Mater Res 813:161-164. https://doi. org/10.4028/www.scientific.net/AMR.813.161

7. Chung C-Y (2019) Integrated optimum layout of conformal cooling channels and optimal injection molding process parameters for optical lenses. Appl Sci 9(20):4341. https://doi.org/10.3390/ app9204341

8. Li CG, Wu Y (2010) Evolutionary Optimization of Plastic Injection Mould Cooling System Layout Design. International Conference on Intelligent System Design and Engineering Application:693696. https://doi.org/10.1109/ISDEA.2010.305

9. Wang Y, Yu K-M, Wang CCL, Zhang Y (2011) Automatic design of conformal cooling circuits for rapid tooling. Comput Aided Des 43(8):1001-1010. https://doi.org/10.1016/j.cad.2011.04.011

10. Zhang Y, Hou B, Wang Q, Li Y, Huang Z (2018) Automatic design of conformal cooling channels in injection molding tooling. IOP Conference Series: Materials Science and Engineering 307:12025. https://doi.org/10.1088/1757-899X/307/1/012025

11. Jahan SA, Wu T, Zhang Y, Zhang J, Tovar A, Elmounayri H (2017) Thermo-mechanical design optimization of conformal cooling channels using Design of Experiments Approach. Procedia Manufacturing 10:898-911. https://doi.org/10.1016/j.promfg. 2017.07.078

12. Rhee B-O, Park C-S, Chang H-K, Jung H-W, Lee Y-J (2010) Automatic generation of optimum cooling circuit for large injection molded parts. Int J Precis Eng Manuf 11(3):439-444. https://doi. org/10.1007/s12541-010-0050-Z

13. Hopmann C, Nikoleizig P (2018) Inverse thermal mold design for injection molds. Int J Mater Form 11(1):113-124. https://doi.org/ 10.1007/s12289-016-1334-3

14. Agazzi A, Sobotka V, LeGoff R, Jarny Y (2013) Optimal cooling design in injection moulding process - a new approach based on morphological surfaces. Appl Therm Eng 52(1):170-178. https:// doi.org/10.1016/j.applthermaleng.2012.11.019

15. Agazzi A, Sobotka V, Le Goff R, Garcia D, Jarny Y (2010) A methodology for the Design of Effective Cooling System in injection Moulding. Int J Mater Form 3(S1):13-16. https://doi.org/10. 1007/s12289-010-0695-2

16. Agazzi A, Sobotka V, Le Goff R, Jarny Y (2013) Uniform cooling and part Warpage reduction in injection molding thanks to the Design of an Effective Cooling System. Key Eng Mater 554-557: 
1611-1618. https://doi.org/10.4028/www.scientific.net/KEM.554557.1611

17. Agazzi A; Garcia D; Jarny Y; Le Goff R; Sobotka V (2018) Method for forming channels in a tool and computer program product that performs such a method. Patentn ${ }^{\circ}$ : WO 2012/168669. B22D 17/20 (2006.01)

18. Nikoleizig P (2017) Inverse thermische Spritzgießwerkzeugauslegung auf basis des lokalen Kühlbedarfs. Dissertation, RWTH Aachen University

19. Hopmann C, Theunissen M, Schneppe T, Schmitz M (2019) Automatic cooling channel design for injection moulds. AIP Conference Proceeding 2139:30004. https://doi.org/10.1063/1. 5121657
20. Hopmann C, Fischer T, Hohlweck T (2019) Simulative and experimental validation of an inversed cooling channel design for injection molds. SPE ANTEC 2019 conference proceedings. Curran associates, Inc. red hook, NY, Vol. 1, pp 339-344

21. Chen S-C, Turng L-S, Ameli A (2019) Advanced injection molding technologies. Hanser, Cincinnati, Ohio

22. Menges G et al (2014) Menges Werkstoffkunde Kunststoffe. Hanser, München

Publisher's note Springer Nature remains neutral with regard to jurisdictional claims in published maps and institutional affiliations. 